\title{
AVALIAÇÃO DE FERTILIZANTES DE EFICIÊNCIA AUMENTADA APLICADOS EM COBERTURA SEM INCORPORAÇÃO NOS PARAMÊTROS MORFOLÓGICOS E PRODUTIVOS NA CULTURA DO CAFÉ ${ }^{1}$
}

\author{
César Ferreira Santos ${ }^{2}$ \\ Sheila Isabel do Carmo Pinto \\ Luciano Eduardo de Carvalho \\ Paulo Otávio Resende Ramalho
}

\begin{abstract}
RESUMO
$\mathrm{O}$ equilíbrio nutricional das plantas é fundamental para se evitar perdas de produtividade. $\mathrm{O} \mathrm{N}$ é o nutriente que proporciona maior resposta em termos de produção na cultura do café, e a fonte nitrogenada mais utilizada é a ureia, sendo sujeita a perdas de $\mathrm{N}$ por volatilização de amônia quando aplicada sem incorporação. Para evitar tais perdas, fontes nitrogenadas de eficiência aumentada têm sido comercializadas como eficientes na redução da volatilização do N. Assim sendo, objetivou-se, com este estudo, avaliar as fontes nitrogenadas aplicadas em cobertura na cultura do café. O trabalho foi desenvolvido no Instituto Federal de Educação, Ciência e Tecnologia de Minas Gerais (IFMG) - Campus Bambuí, em área de cafeeiro do cultivar Rubi, em espaçamento 3 x 0,8m, em um Latossolo Vermelho. O experimento foi realizado em blocos casualizados, com quatro repetições; os tratamentos incluíram sete fontes nitrogenadas, e foram realizadas quatro adubações, por dois anos, com intervalos mensais. As parcelas experimentais foram compostas por 10 plantas; contudo, somente oito foram avaliadas quanto ao diâmetro de copa, altura, número de ramos e produtividade, um mês após a cobertura. Foram analisados o IRCF da clorofila A (IRCA), da clorofila B (IRCB) e a clorofila total (IRCT). O uso dos adubos ureia $+\mathrm{Cu}+\mathrm{B}$ e ureia + NBPT demonstrou, em modificações fisiológicas, que pode gerar um melhor desempenho de crescimento devido a uma maior capacidade fotossintética, posto que o adubo cinco proporcionou aumento na produtividade do cafeeiro.
\end{abstract}

Palavras-chave: Amônia. Clorofila. Produtividade.

\footnotetext{
${ }^{1}$ Como citar este artigo: SANTOS, C. F. et al. Avaliação de fertilizantes de eficiência aumentada aplicados em cobertura sem incorporação nos parâmetros morfológicos e produtivos na cultura do café. ForScience, Formiga, v. 8, n. 1, e00347, jan./jun. 2020. DOI: 10.29069/forscience.2020v8n1.e347.
}

\footnotetext{
${ }^{2}$ Autor para correspondência: César Ferreira Santos, e-mail: agronomocesar.santos@ gmail.com.
} 


\title{
EVALUATION OF INCREASED EFFICIENCY FERTILIZERS APPLIED IN COVERAGE WITHOUT INCORPORATION IN THE MORPHOPHYSIOLOGICAL AND PRODUCTIVE PARAMETERS IN COFFEE CULTURE
}

\begin{abstract}
The nutritional balance of the plants is fundamental to avoid losses of productivity. $\mathrm{N}$ is the nutrient that provides the greatest production response in the coffee crop. The most used nitrogen source is urea, which is subject to $\mathrm{N}$ losses by volatilization of ammonia when applied without incorporation. In order to avoid such losses, nitrogen sources of increased efficiency have been commercialized as efficient in the reduction of $\mathrm{N}$ volatilization. Thus, this work aimed to evaluate the nitrogen sources applied in the coffee crop. The work was developed at the Federal Institute of Education, Science and Technology of Minas Gerais (IFMG) - Bambuí Campus, in a coffee area of the Rubi cultivar, spaced $3 \times 0.8 \mathrm{~m}$ in a Red Latosol. The experiment was performed in a randomized complete block with four replicates, treatments included seven nitrogen sources and four fertilizations were performed for two years with monthly intervals. The experimental plots were composed of 10 plants, however, only eight were evaluated for crown diameter, height, number of branches and productivity one month after coverage. IRCF of chlorophyll A (IRCA), chlorophyll B (IRCB) and total chlorophyll (IRCT) were analyzed. The use of fertilizers urea $+\mathrm{Cu}+\mathrm{B}$ and urea + NBPT translated into physiological modifications that can generate a better growth performance due to a greater photosynthetic capacity, since the fertilizer five provided an increase in coffee productivity.
\end{abstract}

Keywords: Ammonia. Chlorophyll. Productivity.

\section{INTRODUÇÃO}

A cultura do café, no Brasil, merece destaque devido a sua importância na geração de empregos e receitas ao país. Para se ter uma boa produtividade, como em qualquer outra cultura, a sua demanda nutricional deve ser atendida ainda no estágio de desenvolvimento dos frutos, acompanhando a curva de absorção pela cultura (CREWS; PEOPLES, 2005; FUNJINUMA et al., 2009; JIN et al., 2013; AZEEM et al., 2014). Dentre os nutrientes que devem ser aplicados no solo e disponibilizados à cultura, destaca-se o nitrogênio, sendo o primeiro em quantidade e o segundo em exportação pelos grãos (MALAVOLTA, 1986).

No Brasil, existe o conceito entre profissionais e empresários rurais de que, aumentando-se o parcelamento da adubação nitrogenada aumenta-se a eficiência do uso do nitrogênio, o que reduz as perdas, principalmente por lixiviação (COELHO et al., 1992).

Devido ao baixo custo e à alta concentração de nitrogênio, no Brasil, a ureia é o fertilizante nitrogenado mais utilizado, em comparação com sulfato e nitrato de amônio (ROY; HAMMOND, 2004; FERNANDES et al., 2015). No entanto, sob o aspecto agronômico, ForScince, Formiga, v. 8, n. 1, e00347, jan./jun. 2020 
quando aplicada sobre o solo, sem incorporação pela água da chuva ou mecanicamente,ela pode estar sujeita a perdas para a atmosfera na forma de amônia. Em trabalho realizado por Dominghetti et al. (2016), por exemplo, foram encontradas perdas de amônia por volatilização em média de $30 \%$. Em termos da nutrição dessa cultura, tais perdas podem comprometer a disponibilidade de N à planta (FENILLI et al., 2007), refletindo em menor produtividade do cafeeiro.

Nesse sentido, alternativas para a redução das perdas de $\mathrm{N}-\mathrm{NH}_{3}$ por volatilização vêm sendo estudadas, como as boas práticas para uso eficiente de fertilizantes (IFA, 2009; ROCHETTE et al., 2014), a mistura física de fertilizantes, os chamados blendes (TRENKEL, 2010), além do uso de fertilizantes de eficiência aumentada (AZEEM et al., 2014). Dentro destes, enquadram-se a ureia com inibidor de urease, como o NBPT (N-(n-butil) tiofosfóricotriamida) e, aqueles nitrogenados recobertos por uma camada de enxofre elementar $\left(\mathrm{S}^{0}\right)$ e resinas ou polímeros.

Os fertilizantes nitrogenados de eficiência aumentada possuem valor de mercado elevado em comparação aos convencionais (TRENKEL, 2010; CHIEN et al., 2009), sendo a principal limitação o uso em larga escala (AZEEM et al., 2014; TIMILSENA et al., 2015).

Diante do exposto, o objetivo deste trabalho foi estudar a eficiência de fertilizantes nitrogenados convencionais, de liberação lenta e controlada,aplicados em cobertura nos parâmetros morfofisiológicos da cultura do café.

\section{MATERIAL E MÉTODOS}

O experimento foi realizado em uma lavoura de café do cultivar Rubi, com dois anos de idade, sob o espaçamento de 3 x 0,8m, instalada no Departamento de Agricultura do IFMGCampus Bambuí (latitude $18^{\circ} 49^{\prime} 41.02$ ” e longitude $41^{\circ} 58^{\prime}$ 52.07”). O clima da região é subtropical úmido, com temperatura média anual de $22,5^{\circ} \mathrm{C}$ e precipitação pluviométrica média anual de 1426,3 mm. O experimento foi conduzido em um relevo caracterizado como suave, em um LATOSSOLO VERMELHO distroférrico típico, de textura argilosa e com material de origem do tipo calcário (EMBRAPA, 2013).

O experimento foi desenvolvido em blocos casualizados, com sete tratamentos e quatro repetições. No primeiro ano, os tratamentos incluíram a aplicação de $20 \mathrm{~g}$ de $\mathrm{N}$ por planta em cada adubação de cobertura (GUIMARÃES et al., 1999). Já no segundo ano, o plano de adubação foi alterado devido à idade e ao porte da planta, sendo utilizadas $96 \mathrm{~g}$ de N por planta. Os fertilizantes empregados foram: ureia perolada (44\% N), sulfato de amônio $(20 \% \mathrm{~N} ; 22 \%$ 
$\mathrm{S})$, ureia + polímero $(43 \% \mathrm{~N})$, nitrogenado complexo $(29 \% \mathrm{~N} ; 5 \% \mathrm{Ca} ; 9 \% \mathrm{~S} ; 2 \% \mathrm{Mg} ; 0,3 \% \mathrm{~B})$, ureia $+\mathrm{Cu}+\mathrm{B}(44 \% \mathrm{~N} ; 0,16 \% \mathrm{Cu} ; 0,4 \% \mathrm{~B})$, ureia + enxofre elementar $(37 \% \mathrm{~N} ; 16 \% \mathrm{~S})$ e ureia + NBPT (45\% N; com inibidor de urease). O experimento foi constituído por 28 parcelas experimentais compostas por 10 plantas de café, onde somente as oito plantas centrais foram avaliadas. Excluíram-se três linhas em cada extremidade da área, como bordadura, e foram realizadas quatro adubações de cobertura, com intervalos de 30 dias.

Uma semana após cada adubação de cobertura, com os diferentes adubos nitrogenados, foram avaliadas as seguintes características morfológicas e fisiológicas da cultura do cafeeiro: altura de planta, medida desde a superfície do solo até o ápice; diâmetro da copa, determinado no terço médio da planta, medindo-se do colo até o ápice do cafeeiro; número de ramos

plagiotrópicos, contados desde o primeiro ramo da base ao ápice da planta; teor foliar de clorofila, determinado por meio do medidor eletrônico de teor de clorofila, modelo CFL 1030, sendo que os resultados coletados foram obtidos no terceiro par de folhas, sendo três folhas por planta de café; produtividade, determinada por meio da quantidade de café beneficiado produzido pelas oito plantas da área útil e, posteriormente, extrapolado para a área do hectare.

Após tabulados, os dados das variáveis avaliadas foram submetidos à análise de variância e, as médias, comparadas por meio do teste de Tukey a 5\% de probabilidade, utilizando-se o programa estatístico Sisvar (FERREIRA, 2011).

\section{RESULTADOS E DISCUSSÃO}

Não foram observadas diferenças entre as fontes nitrogenadas aplicadas ao café para as variáveis diâmetro de copa, altura e número de ramos plagiotrópicos (NRP) em nenhuma das 4 adubações de cobertura ( $\mathrm{P}>0,05)$ (Tabela 1). A utilização das tecnologias avaliadas, ureia com adição de enxofre, boro, cobre ou inibidor de urease, comercializadas como redutoras da volatilização de $\mathrm{N}_{-} \mathrm{NH}_{3}$ apresentou comportamento semelhante ao da ureia convencional (perolada). No entanto, isso pode ser explicado, em princípio, pela não mensuração das perdas diretas de $\mathrm{N}-\mathrm{NH}_{3}$ por volatilização, ou a redução das perdas pode não ter sido capaz de se refletir em parâmetros morfológicos da planta. 
Tabela 1 - Resumo da análise de variância para as características: diâmetro de copa, altura e número de ramos plagiotrópicos de plantas de café em função de diferentes fontes nitrogenadas utilizadas na

\begin{tabular}{|c|c|c|c|c|}
\hline Adubação & $\begin{array}{l}\text { Fonte de } \\
\text { variação }\end{array}$ & $\begin{array}{l}\text { Diâmetro de } \\
\text { copa }(\mathrm{cm})\end{array}$ & Altura $(\mathrm{cm})$ & NRP \\
\hline $1^{\mathrm{a}}$ adubação & $\begin{array}{c}\text { Fonte Nitrogenada } \\
\text { CV }(\%) \\
\text { Média }\end{array}$ & $\begin{array}{l}0,08^{\mathrm{NS}} \\
20,8 \\
82,2\end{array}$ & $\begin{array}{c}0,250^{\mathrm{NS}} \\
10,8 \\
73,67\end{array}$ & $\begin{array}{c}0,06^{\mathrm{NS}} \\
21,19 \\
36,0\end{array}$ \\
\hline $2^{\mathrm{a}}$ adubação & $\begin{array}{c}\text { Fonte Nitrogenada } \\
\text { CV }(\%) \\
\text { Média }\end{array}$ & $\begin{array}{c}0,214^{\mathrm{NS}} \\
17,7 \\
82,2\end{array}$ & $\begin{array}{c}0,145^{\mathrm{NS}} \\
10,5 \\
81,12\end{array}$ & $\begin{array}{c}0,47^{\mathrm{NS}} \\
21,7 \\
40,0\end{array}$ \\
\hline $3^{\mathrm{a}}$ adubação & $\begin{array}{c}\text { Fonte Nitrogenada } \\
\text { CV }(\%) \\
\text { Média }\end{array}$ & $\begin{array}{c}0,06^{\mathrm{NS}} \\
17,4 \\
90,5\end{array}$ & $\begin{array}{c}0,399^{\mathrm{NS}} \\
10,8 \\
88,3\end{array}$ & $\begin{array}{c}0,106^{\mathrm{NS}} \\
19,6 \\
43,7\end{array}$ \\
\hline $4^{\mathrm{a}}$ adubação & $\begin{array}{c}\text { Fonte Nitrogenada } \\
\text { CV }(\%) \\
\text { Média }\end{array}$ & $\begin{array}{c}0,09^{\mathrm{NS}} \\
15,9 \\
97,5\end{array}$ & $\begin{array}{c}0,07^{\mathrm{NS}} \\
10,7 \\
93,0\end{array}$ & $\begin{array}{c}0,570^{\mathrm{NS}} \\
19,5 \\
47,6\end{array}$ \\
\hline
\end{tabular}

Na Tabela 2, são apresentados os resultados da análise de variância para as variáveis clorofila A, B, total e para a relação entre as clorofilas A e B. Podem-se perceber diferenças significativas nos níveis de clorofila A após a segunda adubação de cobertura; clorofila B, após a terceira e quarta adubações; clorofila total, após a segunda adubação; e na relação clorofila $\mathrm{A} / \mathrm{B}$, após a terceira e quarta adubações. Estes resultados contradizem os encontrados por Chagas et al. (2016), que não encontraram diferenças relevantes no teor de clorofila na cultura do café adubada com diferentes fontes de N. Os dados que não apresentaram diferenças estatísticas não estão na tabela.

Tabela 2 - Resumo da análise de variância para as características avaliadas na cultura do café em função de diferentes fontes nitrogenadas utilizadas na adubação de cobertura

(continua)

\begin{tabular}{cccccc}
\hline Medição & FV & Clorofila A & Clorofila B & $\begin{array}{c}\text { Clorofila } \\
\text { Total }\end{array}$ & Relação A/B \\
& & & & $0,001^{*}$ & $0,141^{\text {NS }}$ \\
\hline Após 2 ${ }^{\text {a adubação }}$ & Fonte & $0,000^{*}$ & $0,316^{\mathrm{NS}}$ & 8,2 & 15,9 \\
& CV $(\%)$ & 6,9 & 16,2 & 69,6 & 1,93
\end{tabular}


(conclusão)

$\begin{array}{cccccc}\text { Após 3 } 3^{\mathrm{a}} \text { adubação } & \text { Fonte } & 0,207^{\mathrm{NS}} & 0,030^{*} & 0,573^{\mathrm{NS}} & 0,000^{*} \\ & \text { CV (\%) } & 4,9 & 9,9 & 5,7 & 10,8 \\ & \text { Média } & 45,6 & 28,6 & 74,3 & 1,62 \\ & & & & & \\ \text { Após 4 } & & & & \\ & \text { Fonte } & 1,111^{\mathrm{NS}} & 0,040^{*} & 0,105^{\mathrm{NS}} & 0,031^{*} \\ & \text { CV (\%) } & 4,7 & 10,8 & 6,1 & 10,7 \\ & \text { Média } & 45,9 & 29,4 & 75,4 & 1,60\end{array}$

*Significativo pelo teste de $\mathrm{F}$ a $5 \%$ de probabilidade; ${ }^{\mathrm{NS}}$ Não significativo pelo teste $\mathrm{F}$ a $5 \%$ de probabilidade. FV: Fator de variação. Fonte: Fonte nitrogenada. CV: Coeficiente de variação Fonte: Autores (2019).

Nas Figuras 1, 2 e 3, são apresentadas as médias obtidas para os índices relativos de clorofila A (IRCA), B (IRCB), total (IRCT) e relação IRCA/IRCB em função das diferentes fontes nitrogenadas aplicadas no $2^{\circ}, 3^{\circ}$ e $4^{\circ}$ parcelamentos da adubação nitrogenada de cobertura no café.

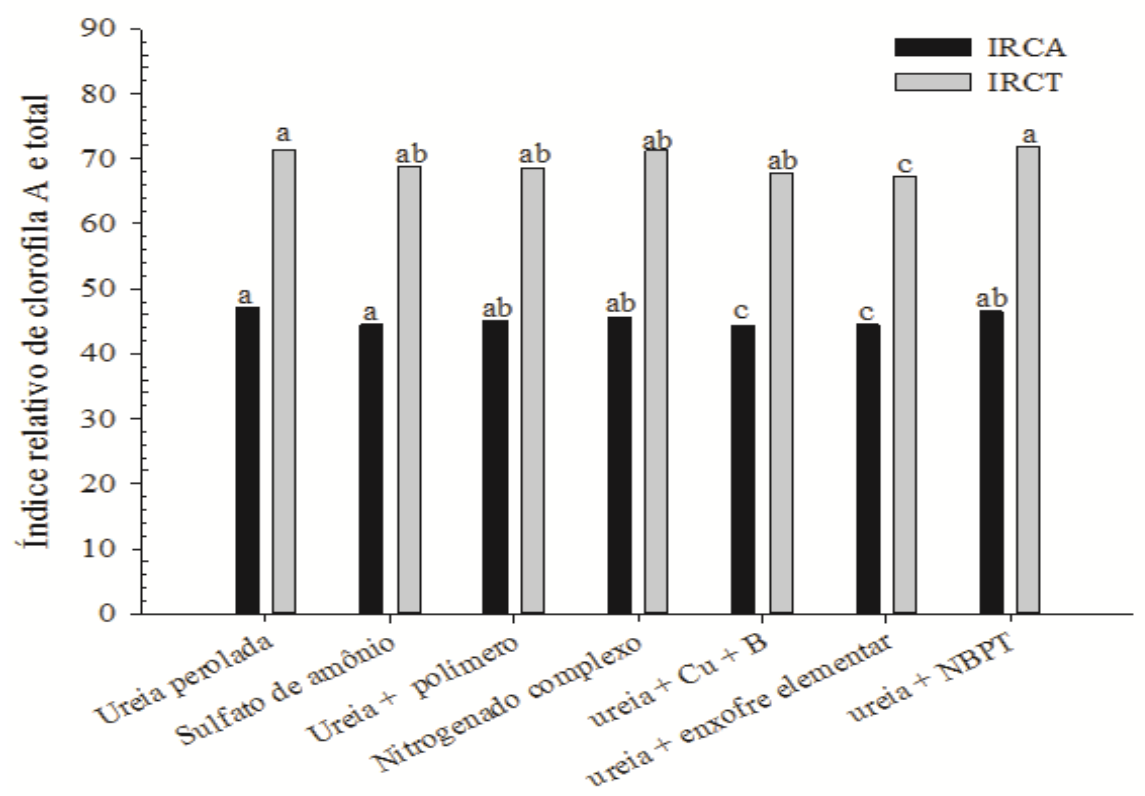

Figura 1 - Índices de clorofila A (IRCA) e clorofila total (IRCT), medidos após o $2^{\circ}$ parcelamento da adubação nitrogenada em cobertura no café. *Médias seguidas de mesma letra, nas barras, não diferem entre si pelo teste de Tukey a $5 \%$ de probabilidade

Fonte: Autores (2019). 
A adubação-controle, utilizando a ureia perolada, apresentou resultados expressivos para a segunda medição nos parâmetros clorofila A e clorofila total. Porém o fertilizante ureia + NBPT obteve os mesmos níveis do controle com ureia.

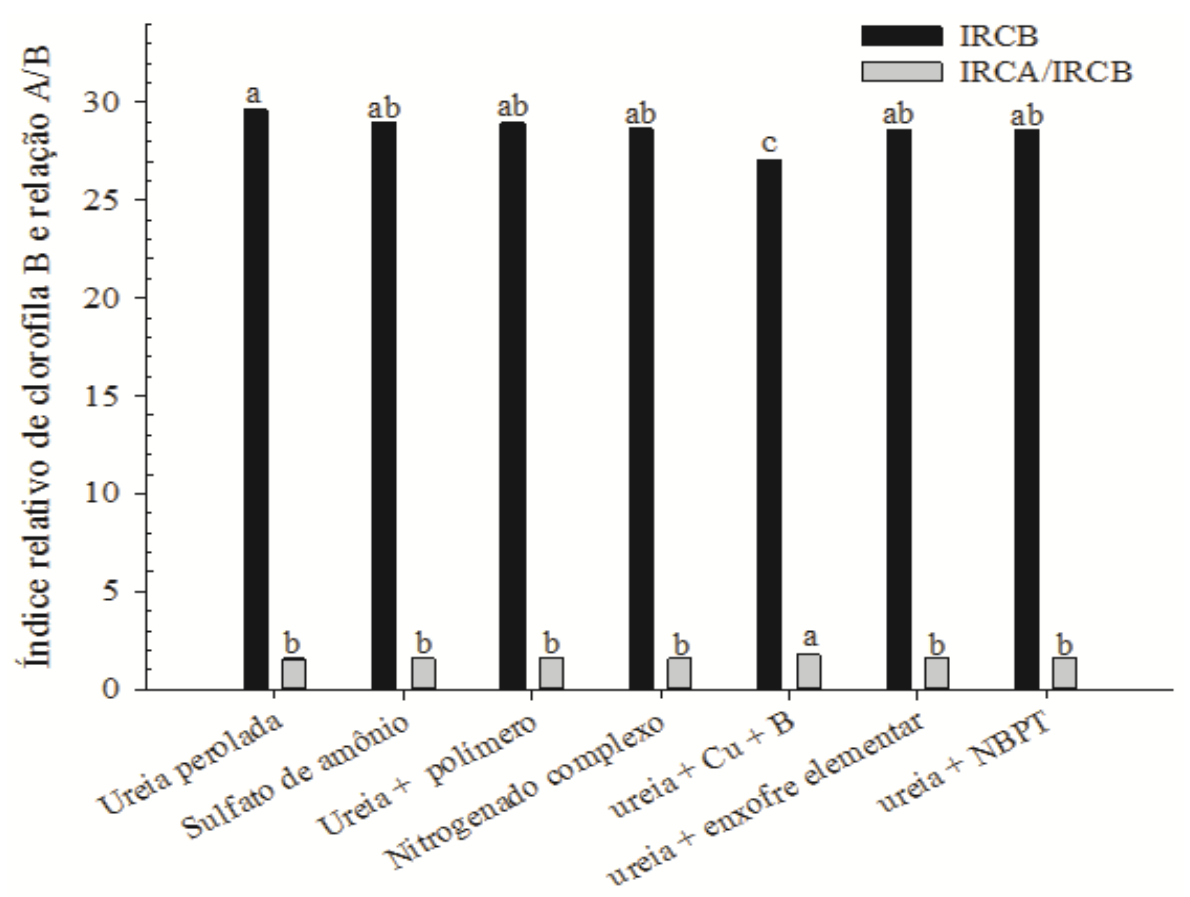

Figura 2 - Índices de clorofila B (IRCB) e relação clorofila A e B (IRCA/IRCB), medidos após o $3^{\circ}$ parcelamento da adubação nitrogenada em cobertura no café. *Médias seguidas de mesma letra, nas barras, não diferem entre si pelo teste de Tukey a $5 \%$ de probabilidade Fonte: Autores (2019).

Para a terceira medição, foram observadas diferenças consideráveis nos parâmetros clorofila $\mathrm{B}$ e relação clorofila $\mathrm{A} / \mathrm{B}$, onde a ureia perolada e ureia $+\mathrm{Cu}+\mathrm{B}$ destacaram-se, respectivamente, em relação às demais fontes nitrogenadas.

Já na quarta medição, o tratamento ureia perolada apresentou maior índice de clorofila $\mathrm{A}$, enquanto os fertilizantes ureia $+\mathrm{Cu}+\mathrm{B}$ e ureia + NBPT destacaram-se entre as demais fontes testadas para relação clorofila A/B e clorofila B, respectivamente. 


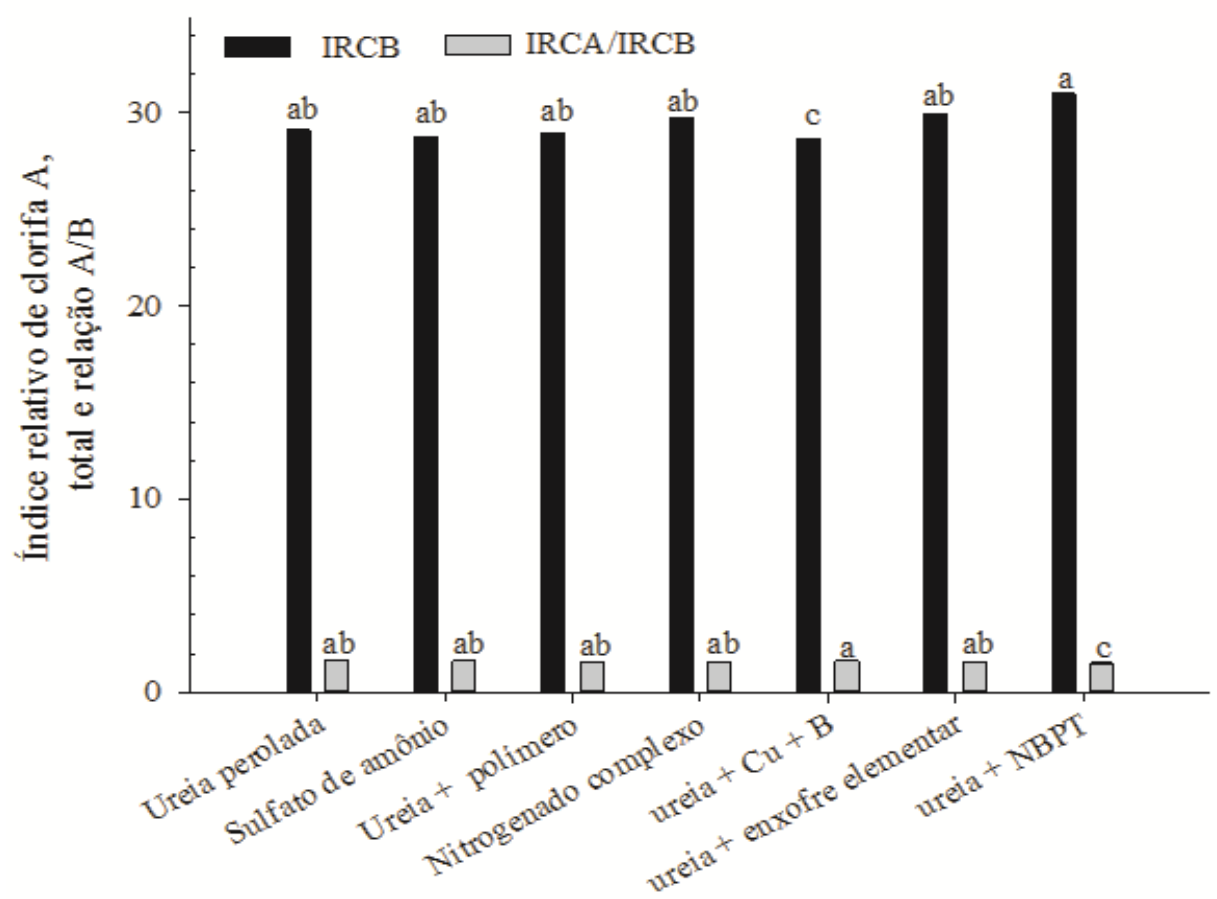

Figura 3 - Índices de clorofila B (IRCB) e relação clorofila A e B (IRCA/IRCB), medidos após a $4^{\circ}$ parcelamento da adubação nitrogenada em cobertura no café. *Médias seguidas de mesma letra, nas barras, não diferem entre si pelo teste de Tukey a $5 \%$ de probabilidade Fonte: Autores (2019).

A relação clorofila $\mathrm{A} / \mathrm{B}$ mais elevada garante um melhor transporte de energia e, consequentemente, uma maior eficiência fotossintética (TAIZ; ZEIGER, 2013). Dessa forma, a ureia $+\mathrm{Cu}+\mathrm{B}$ parece ter uma influência positiva em relação aos níveis de clorofila do cafeeiro. Apesar das diferenças não serem muito expressivas, fisiologicamente, pequenas diferenças podem ser expressas em ganhos em termos de produção (TAIZ; ZEIGER, 2013).

A produtividade do cafeeiro foi influenciada significativamente pelos fertilizantes aplicados em cobertura $(\mathrm{P}<0,05)$ (Tabela 4$)$.

Tabela 4 - Resumo da análise de variância para a produção do cafeeiro em função de diferentes fontes nitrogenadas

\begin{tabular}{cc}
\hline FV & Produtividade \\
\hline Tratamentos & $0,0033^{*}$ \\
Bloco & $0,1340^{\mathrm{NS}}$ \\
Média & 16.5 \\
CV $(\%)$ & 23
\end{tabular}

\footnotetext{
*Significativo pelo teste $\mathrm{F}$ a $5 \%$ de probabilidade; ${ }^{\mathrm{NS}}$ Não significativo pelo teste $\mathrm{F}$ a $5 \%$ de probabilidade. $\mathrm{FV}$ : Fator de variação. Fonte: Fonte nitrogenada. CV: Coeficiente de variação Fonte: Autores (2019)
} 
$\mathrm{Na}$ Figura 4, são apresentados os resultados do teste de média para as diferentes fontes nitrogenadas aplicadas na adubação de cobertura na cultura do café. Pode-se observar que a ureia $+\mathrm{NBPT}$, ureia $+\mathrm{Cu}+\mathrm{B}$ e a ureia + enxofre elementar, nitrogenado complexo e ureia perolada destacaram-se entre as fontes nitrogenadas avaliadas, proporcionando maior produtividade à cultura.

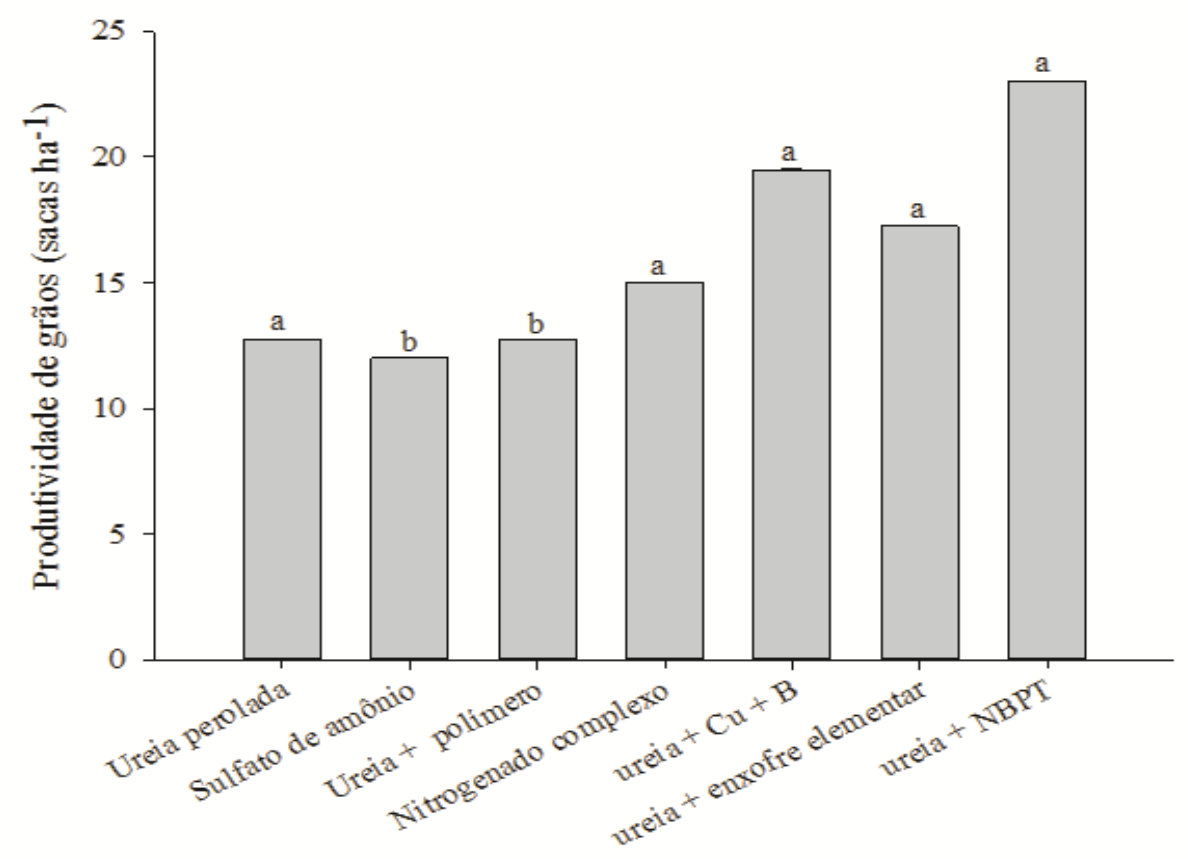

Figura 4 - Produtividade do cafeeiro no ano agrícola, após adubação nitrogenada. *Médias seguidas de mesma letra, nas barras, não diferem entre si pelo teste de Tukey a 5\% de probabilidade

Fonte: Autores (2019).

Chagas et al. (2016), em experimento conduzido em lavoura cafeeira em produção há seis anos, não observaram diferenças entre as fontes de nitrogênio aplicadas, o que se deve, provavelmente, às reservas de nutrientes acumuladas na planta ao longo do cultivo, além do teor de matéria orgânica desses solos. Isso porque solos recém-cultivados com a cultura do café, como salientado anteriormente, possuem maior resposta às adubações.

A maior resposta em produtividade da ureia tratada com NBPT se deve ao fato de esse aditivo inibir a atividade da enzima urease, uma vez que, dessa forma, o fertilizante permanece por maior tempo na forma amídica, retardando a hidrólise da ureia, podendo, consequentemente, ser incorporada pela água da chuva (SOUZA et al., 2017).

No caso da ureia recoberta com $\mathrm{B}$ e $\mathrm{Cu}$, esses nutrientes estabilizam a ureia, mantendo o nitrogênio na forma amídica por um período maior de tempo, como na ureia NBPT, causando uma redução na atividade da urease (SOARES et al., 2012). Sendo a eficiência desse fertilizante ForScince, Formiga, v. 8, n. 1, e00347, jan./jun. 2020 
dependente das condições que causam o rompimento do revestimento, tal comportamento pôde ser constatado neste trabalho.

A ureia revestida com enxofre reduz perdas por volatilização e aumenta os ganhos em produtividade das culturas, devido ao enxofre elementar promover uma proteção física quanto à entrada de água no interior do grânulo de ureia, evitando a liberação do nitrogênio para o solo (TRENKEL, 2010). A liberação do nutriente para a solução se dá por difusão, de acordo com a lei de Fick (AZEEM et al., 2014).

Segundo Khan et al. (2015), os fertilizantes recobertos com enxofre, micronutrientes e polímeros, podem reduzir a rápida liberação do $\mathrm{N}$ para o solo, como também fornecer outros nutrientes contidos no recobrimento.

No trabalho conduzido por Chagas et al. (2016), embora não tenham reparado diferenças em produtividade do cafeeiro com a aplicação de distintas fontes, foram encontradas diferenças em rendimento, ou seja, a quantidade de café da roça para equivaler a uma saca de $60 \mathrm{~kg}$, onde o melhor rendimento foi obtido por um blend entre ureia convencional $30 \%$ e ureia revestida com enxofre, aplicado em uma única vez a $70 \%$ da dose recomendada.

\section{CONCLUSÃO}

Nas condições edafoclimáticas em que o experimento foi conduzido, não foram encontradas diferenças entre as diversas fontes nitrogenadas utilizadas sobre o diâmetro de copa, a altura das plantas ou o número de ramos plagiotrópicos emitidos pelas plantas de café em nenhuma das quatro avaliações realizadas mensalmente após cada adubação de cobertura.

$\mathrm{O}$ uso dos adubos nitrogenados ureia $+\mathrm{Cu}+\mathrm{B}$ e Ureia + NBPT foi traduzido em modificações fisiológicas que podem gerar um melhor crescimento devido a uma maior capacidade fotossintética, mostrada pelo índice relativo da clorofila foliar (IRCF). Além disso, os fertilizantes ureia $+\mathrm{NBPT}$, ureia $+\mathrm{Cu}+\mathrm{B}$, nitrogenado complexo e ureia $+\mathrm{S}$ demonstraram ser a forma mais eficiente para garantir o aumento da produtividade do cafeeiro, justificando o seu potencial de uso.

\section{REFERÊNCIAS}

AZEEM, B. et al. Review on materials \& methods to produce controlled release coated urea fertilizer. Journal of Controlled Release, Amsterdã, v. 18, p.11-21, 2014. 
CHAGAS, W. F. T. et al. Ammonia volatilization from blends with stabilized and controlledreleased urea in the coffee system. Ciência e Agrotecnologia, Lavras, v. 40, n. 5, p. 497-509, 2016.

CHIEN, S.-H.; PROCHNOW, L. I.; CANTARELLA, H. Recent developments of fertilizer production and use to increase nutrient efficiency and minimize environmental impacts. Advances in Agronomy, Amsterdã, v. 102, p. 261-316, 2009.

COELHO, A. M. et al. Doses e métodos de aplicação de fertilizantes nitrogenados na cultura do milho sob irrigação. Revista Brasileira de Ciência do Solo, Viçosa, v. 16, p. 61-67, 1992.

CREWS, T. E.; PEOPLES, M. B. Can the synchrony of nitrogen supply and crop demand be improved in legume and fertilizer base agroecosystems? A review. Nutrient Cycling in Agroecosystem, Switzerland, v. 72, p. 101-120, 2005.

DOMINGHETTI, A. W. et al. Nitrogen loss by volatilization of nitrogen fertilizers applied to coffee orchard. Ciência e Agrotecnologia, Lavras, v. 40, n. 2, p. 1-11, 2016.

EMPRESA BRASILEIRA DE PESQUISA AGROPECUÁRIA, Sistema brasileiro de classificação de solos. Rio de Janeiro: EMBRAPA, 2013. 353p.

FENILLI, T. A. B. et al. Volatilization of ammonia derived from fertilizer and its reabsorption by coffee plants. Communications in Soil Science and Plant Analysis, New York, v. 38, p. 1741-1751, 2007.

FERNANDES, J. C. et al. Sources and rates of nitrogen fertilizer used in Mombasa guineagrass in the Brazilian Cerrado region. African Journal of Agricultural Research, Lagos, v. 10, n. 10, p. 1031-1042. 2015.

FERREIRA. D. F. Sisvar: um sistema computacional de análise estatística. Ciência e Agrotecnologia, Lavras, v. 35, n. 6, p. 1039-1042. 2011.

FUNJINUMA, R.; BALSTER, N. J.; NORMAM, J. M. An improved model of nitrogen release for surfasse-applied urea controlled-released fertilizer. Soil Science Society of American Journal, Madison, v. 73, p. 2043-2050, 2009.

GUIMARÃES, P. T. G. et al. Recomendações para o uso de corretivos e fertilizantes em Minas Gerais, 5a Aproximação. Viçosa: CFSEMG, 1999. 289-302 p.

IFA. The International Fertilizer industry Association. The global "4R" nutrient stewardship framework for developing and delivering fertilizer best management practices, p. 10, 2009.

JIN, S. et al. Preparation and properties of a degradable interpenetrating polymer networks based on starch with water retention, amelioration of soil, and slow release of nitrogen and phosphorus fertilizer. Journal of applied Polymer Science. New York, v. 128, p. 407-415, 2013. 
KHAN, A. Z. et al. Effects of sulfur and urease coated controlled release urea on dry matter yield, n uptake and grain quality of rice. The Journal of Animal \& Plant Sciences, Lahore, v. 25, n. 3, p. 679-685, 2015.

MALAVOLTA, E. Nutrição, adubação e calagem para o cafeeiro. Piracicaba,1986. p. 136274.

ROCHETTE, P. et al. Ammonia volatilization an nitrogen retention: how deep to incorporate urea? Journal Environmental Quality, Madison, v. 42, n. 6, p. 1635-1642, 2014.

ROY, A.; HAMMOND, L. In: MOSIER, A. R.; SYERS J. K.; FRENCY, J. R. (ed.). Challenges and opportunities for the fertilizer industry. Agriculture and the nitrogen cycle. Washington, 2004, p. 231-241.

SOARES, J. R.; CANTARELLA, H.; MENEGALE, M. L. de C. Ammonia volatilization losses from surface applied urea with urease and nitrification inhibitors. Soil Biology and Biochemistry, Brisbane, v. 52, p. 82-89, 2012.

SOUZA, T. L. de et al. Ammonia and carbon dioxide emissions by stabilized conventional nitrogen fertilizers and controlled release in corn crop. Ciência e Agrotecnologia, Lavras, v. 41, n. 5, p. 494-510, 2017.

TAIZ, L.; ZEIGER, E. Fisiologia vegetal. 5. ed. Porto Alegre: Artmed, 2013. 918 p.

TIMILSENA, Y. P. et al. Enhanced efficiency fertilizers: a review of formulation and nutrient release patterns. Journal of the Science and Food Agriculture, New York, v. 95, n. 6, p. 1131-1142, 2015.

TRENKEL, M. E. Slow- and controlled-release and stabilized fertilizers. An option for enhancing nutrient use efficiency in agriculture. International Fertilizer Industry Association, p. 163, 2010.

\section{DADOS DOS AUTORES}

\section{Nome: César Ferreira Santos}

E-mail: agronomocesar.santos@gmail.com

Currículo Lattes: http://lattes.cnpq.br/1071470051957403

Doutorando em Ciência do Solo pela Universidade Federal de Lavras (UFLA). Atualmente é Técnico do Instituto Federal de Educação, Ciência e Tecnologia do Sudeste de Minas Gerais Campus Barbacena. Atua nas áreas de Fertilidade do Solo.

\section{Nome: Sheila Isabel do Carmo Pinto}

E-mail: sheila.isabel@ifmg.edu.br

Currículo Lattes: http://lattes.cnpq.br/7496649581948459

Doutora em Ciência do Solo pela Universidade Federal de Lavras (UFLA). Atualmente é professora do Instituto Federal de Educação, Ciência e Tecnologia de Minas Gerais - Campus Bambuí. Atua nas áreas de Fertilidade do Solo e Nutrição Mineral de Plantas. 


\section{Nome: Luciano Eduardo de Carvalho}

E-mail: lucianoecarvalho@hotmail.com

Currículo Lattes: http://lattes.cnpq.br/9957743380447196

Engenheiro Agrônomo formado pelo Instituto Federal de Educação, Ciência e Tecnologia de Minas Gerais - Campus Bambuí.

\section{Nome: Paulo Otávio Resende Ramalho}

E-mail: pauloagrokz@hotmail.com

Engenheiro Agrônomo formado pelo Instituto Federal de Educação, Ciência e Tecnologia de Minas Gerais - Campus Bambuí. 